\title{
Deficiency of Tfh Cells and Germinal Center in Deceased COVID-19 Patients $^{*}$
}

\author{
Ya-qi DUAN ${ }^{1,2 \dagger}$, Ming-hui XIA ${ }^{3 \dagger}$, Liang REN ${ }^{4 \dagger}$, Yan-fang ZHANG ${ }^{5 \dagger}$, Qi-lin $\mathrm{AO}^{1,2}$, San-peng XU1 ${ }^{1}$, Dong KUANG ${ }^{1}$, Qian LIU ${ }^{4}$, \\ Bing YAN ${ }^{5}$, Yi-wu ZHOU ${ }^{4}$, Qian $\mathrm{CHU}^{6}$, Liang LIU ${ }^{4 \#}$, Xiang-Ping YANG ${ }^{3 \#}$, Guo-ping WANG ${ }^{1,2 \#}$ \\ ${ }^{1}$ Institute of Pathology, Tongji Hospital, Tongji Medical College, Huazhong University of Science and Technology, Wuhan \\ 430030, China \\ ${ }^{2}$ Department of Pathology, School of Basic Medicine, Tongji Medical College, Huazhong University of Science and Technology, \\ Wuhan 430030, China \\ ${ }^{3}$ Department of Immunology, School of Basic Medicine, Tongji Medical College, Huazhong University of Science and \\ Technology, Wuhan 430030, China \\ ${ }^{4}$ Department of Forensic Medicine, Tongji Medical College, Huazhong University of Science and Technology, Wuhan 430030, \\ China \\ ${ }^{5}$ State Key Laboratory of Virology, Wuhan Institute of Virology, Center for Biosafety Mega-Science, Chinese Academy of \\ Sciences, Wuhan 430071, China \\ ${ }^{6}$ Department of Oncology, Tongji Hospital, Tongji Medical College, Huazhong University of Science and Technology, Wuhan \\ 430030, China
}

(C) Huazhong University of Science and Technology 2020

\begin{abstract}
Summary: The COVID-19 pandemic caused by SARS-CoV2 is characterized by a remarkable variation in clinical severity ranging from a mild illness to a fatal multi-organ disease. Understanding the dysregulated human immune responses in the fatal subjects is critical for management of COVID-19 patients and the pandemic. In this study, we examined the immune cell compositions in the lung tissues and hilar lymph nodes using immunohistochemistry on 6 deceased COVID-19 patients and 4 focal organizing pneumonia (FOP) patients who underwent lung surgery and served as controls. We found a dominant presence of macrophages and a general deficiency of $\mathrm{T}$ cells and B cells in the lung tissues from deceased COVID-19 patients. In contrast to the FOP patients, Tfh cells and germinal center formation were largely absent in the draining hilar lymph nodes in the deceased COVID-19 patients. This was correlated with reduced IgM and IgG levels compared to convalescent COVID-19 patients. In summary, our data highlight a defect of germinal center structure in deceased COVID-19 patients leading to an impaired humoral immunity. Understanding the mechanisms of this deficiency will be one of the key points for the management of this epidemic.
\end{abstract}

Key words: COVID-19; immune responses; germinal center; $\mathrm{T}$ follicular helper cells

The COVID-19 worldwide pandemic has been characterized by a remarkable variation in clinical severity ranging from a mild coryzal illness to a fatal multi-organ disease ${ }^{[1,2]}$. Despite the identification of

Ya-qi DUAN, E-mail: yqduan@hust.edu.cn; Ming-hui XIA, E-mail: minghuixia@hust.edu.cn; Liang REN, E-mail: 36918280@qq.com; Yan-fang ZHANG, E-mail: zhangyf@, wh.iov.cn

${ }^{\dagger}$ These authors contributed equally this work.

\#Corresponding authors, Liang LIU, E-mail: liuliang@mails. tjmu.edu.cn; Xiang-ping YANG, E-mail: yangxp@hust.edu. cn; Guo-ping WANG, E-mail: wanggp@hust.edu.cn.

*The study was funded by grants from the Special R\&D Program of Ministry of Science and Technology (No. 2019YFC1316203), Ministry of Science and Technology (No. 2020YFC0844700), Clinical Foundation of Tongji Hospital (No. XXGZBDYJ010). many risk factors for patient survival including age and pre-existing comorbidities like hypertension, diabetes and chronic kidney disease, our understanding of the patho-physiology that explains these risk factors is incomplete.

SARS-CoV2, along with the related SARS$\mathrm{CoV}$, uses the ACE2 enzyme as a receptor for the infection of the respiratory epithelial cells ${ }^{[3,4]}$. Acute respiratory disease syndrome (ARDS) is the principle cause of death in patients with COVID-19 although multiple organ failure has been noted ${ }^{[2,5]}$. It appears the morbidity is associated with an aberrant inflammatory immune response, as Tocilizumab has been effective in clinical trials $s^{[6,7]}$. Paradoxically, critically ill patients display a significant peripheral lymphopenia, which is associated with a poor prognosis ${ }^{[8,9]}$. Characterization of compositions of the immune cells within the 
lung tissues and draining hilar lymph nodes from the postmortem specimens might provide valuable insights on how the immune responses in the deceased patients were dysregulated and offer new strategies for treatment.

\section{MATERIALS AND METHODS}

\subsection{Patients}

This study was in accordance with regulations issued by the National Health Commissions of China (KY-2020-15.01) and approved by the Ethics Committee of Tongji Hospital, Tongji Medical College, Huazhong University of Science and Technology, Wuhan, China (No. TJ-IRB20200386). The written consents of autopsy of 6 deceased COVID-19 patients who had been admitted in Jinyintan Hospital, Wuhan, China were obtained from the next of kin prior to the autopsies. Four control patients with focal organizing pneumonia (FOP) who underwent lung surgery were involved in a separate study (No. TJ-IRB20200209) and approved by Ethics Committee of Tongji Hospital, Tongji Medical College, Huazhong University of Science and Technology, Wuhan, China. At the time of enrollment in the initial study, all individuals provided informed consent that their samples could be used for future studies. For the antibody detection, samples of sera were collected from 8 convalescent COVID-19 patients who had been admitted in Tongji Hospital, Wuhan, China and written consents were obtained. Diagnosis of COVID-19 was based on the New Coronavirus Pneumonia Prevention and Control Program (5th edition) published by the National Health Commission of China. All the patients were laboratory-confirmed positives for SARS-CoV-2 by the quantitative RT-PCR (qRT-PCR) on throat swab samples.

\subsection{Immunohistochemistry and Analysis}

The histopathology and pathological characteristics of lung tissues and hilar lymph nodes were analyzed by H\&E staining. Briefly, paraffin-embedded tissue blocks were cut into 3- $\mu \mathrm{m}$ slices and mounted onto poly-lysine-coated glass slides, tissues were incubated with hematoxylin for $5 \mathrm{~min}$, after $1 \mathrm{~min}$ of dehydration by $100 \%$ alcohol, sections were further treated with eosin for $30 \mathrm{~s}$. The results were viewed using a light microscope (Olympus). The immunohistochemistry was performed according to standard procedures. Briefly, specimens were fixed in $4 \%$ formaldehyde and embedded in paraffin. Deparaffinized slides were antigen retrieved in citrate buffer $(\mathrm{pH}$ 6.4) and endogenous peroxidase (HRP) activity was blocked by treating the sections with $3 \%$ hydrogen peroxide in methanol. Indirect immunohistochemistry was performed with species-specific biotinylated secondary antibodies followed by avidin-horseradish peroxidase.
The following primary antibodies were used: antiCD68, anti-CD3, anti-CD4, anti-CD8, anti-CD20, antiCD38, anti-PD-1. Antibody information is presented in table 1. Finally, sample sections were viewed using a light microscope (Olympus). ImageJ software was used to quantify the number of infiltrated immune cells.

Table 1 Antibody information

\begin{tabular}{llll}
\hline $\begin{array}{l}\text { Primary } \\
\text { antibodies }\end{array}$ & Clone ID & Dilution & Vendor \\
\hline CD68 & KP1 & $1: 400$ & DAKO \\
CD3 & F7.2.38 & $1: 400$ & DAKO \\
CD4 & UMAB64 & Ready to use & ZSGB-BIO \\
CD8 & SP16 & Ready to use & ZSGB-BIO \\
CD20 & L28 & $1: 400$ & DAKO \\
CD38 & SPC32 & Ready to use & ZSGB-BIO \\
PD-1 & UMAB199 & Ready to use & ZSGB-BIO \\
\hline
\end{tabular}

\subsection{Quantification of Germinal Center}

Germinal center (GC) number quantification and the ratio of GC number to total follicle numbers in hilar lymph nodes were determined by H\&E staining and histology morphology. The data were evaluated by two independent pathologists.

\subsection{Detection of IgG and IgM against SARS-CoV-2}

Serum samples of convalescentCOVID-19 patients shortly before discharge and deceased COVID-19 patients shortly before death were collected. All serum samples were inactivated at $56^{\circ} \mathrm{C}$ for $30 \mathrm{~min}$ and stored at $-80^{\circ} \mathrm{C}$ before testing. The amounts of $\operatorname{IgG}$ against nucleocapsid $(\mathrm{N})$ protein and IgM against receptorbing domain (RBD) of the spike (S) protein of SARS$\mathrm{CoV}-2$ in plasma samples were tested using ELISA kits (Zhuhai Lizhu Reagent Co., Ltd., China) respectively according to the manufacture's description. All tests were performed under strict biosafety conditions.

\subsection{Statistical analysis}

Statistical analysis was performed using GraphPad Prism software. Qualitative variables were expressed as numbers. Statistical significance was determined by Student's $t$ test. $P$ values less than 0.05 were considered significant.

\section{RESULTS}

To gain insight into the human immune responses during a fatal SARS-CoV2 infection, we performed postmortem autopsy studies of the immune cell compositions within the lung tissues and hilar lymph nodes using immunohistochemistry on 6 deceased COVID-19 patients, and 4 FOP patients who underwent lung surgery served as controls. The demographic information of all patients is presented in table 2 . The average hospitalization day of the 6 COVID-19 patients (average 71.5 years old) was 25 days.

Histologically, the lung tissues of the deceased COVID-19 patients exhibited edema, proteinaceous 


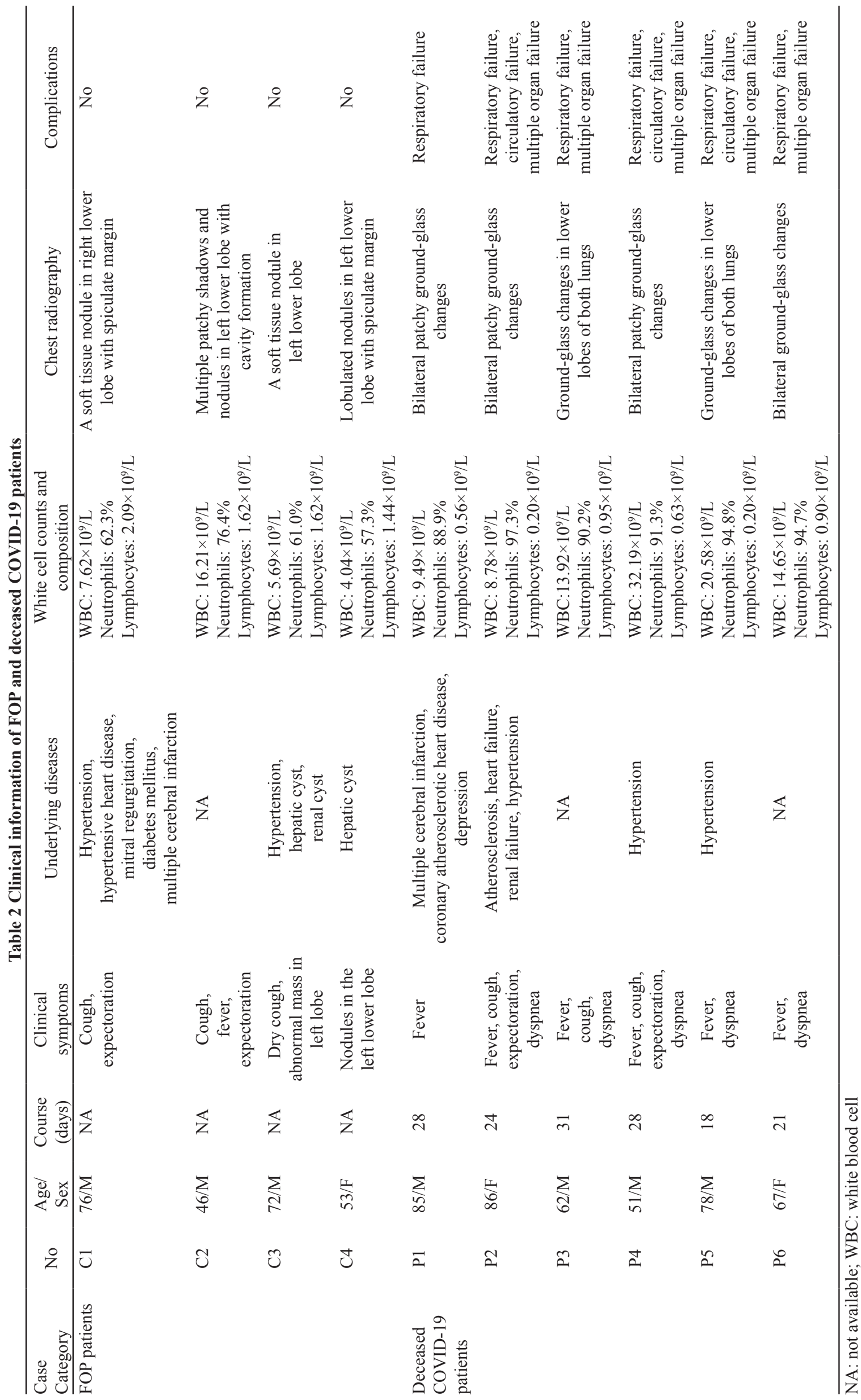


exudate forming hyaline membrane, scattered interstitial lymphocyte infiltration and variable amount of intraalveolar macrophages with pneumocyte hyperplasia (fig. 1A). In comparison to the FOP patients showing presence of $\mathrm{CD} 68^{+}$macrophages, as well as $\mathrm{CD}^{+}$, $\mathrm{CD} 4^{+}$, and $\mathrm{CD} 8^{+} \mathrm{T}$ cells, $\mathrm{CD} 20^{+} \mathrm{B}$ cells and $\mathrm{CD} 38^{+}$ plasma cells, the dominant cell population within the lung tissues from the deceased COVID-19 patients was $\mathrm{CD} 68^{+}$macrophages (fig.1A), while the percentages of $\mathrm{CD}^{+}, \mathrm{CD}^{+}, \mathrm{CD}^{+} \mathrm{T}$ cells, $\mathrm{CD} 20^{+} \mathrm{B}$ cells and $\mathrm{CD} 38^{+}$ plasma cells were significantly reduced compared to FOP patients (fig. 1B). This observation indicates a dominant innate immune response within the lung tissues of non-survival COVID-19 patients.

Activated B cells undergo clonal expansion and selection within the secondary lymphoid organs, forming a distinct microanatomy structure named GC, which is necessary for antibody diversification and affinity maturation, a process vitally important for mounting appropriate humoral immunity ${ }^{[10,11]}$. In contrast to the control patients who had GCs and presence of PD- $1^{+}$cells (human Tfh) within the GCs, there were few GCs or Tfh cells within the hilar lymph nodes of deceased COVID-19 patients (fig. 2A). T cells were more coalesced within the control patients but more dispersed within the hilar lymph nodes of deceased COVID-19 patients (fig. 2A). The CD20+ $\mathrm{B}$ cells were clustered and coalesced as noticeable GCs in the FOP control patients, but they were evenly dispersed in the COVID-19 lymph nodes (fig. 2A). Similarly, CD $38^{+}$plasma cells were found in both $\mathrm{GC}$ and non-GC areas in FOP samples whereas those cells were evenly dispersed in the COVID-19 lymph nodes (fig. 2A). Quantifications of GC number per slide area and ratios of GCs among follicular number in both groups showed significant reduction within
A
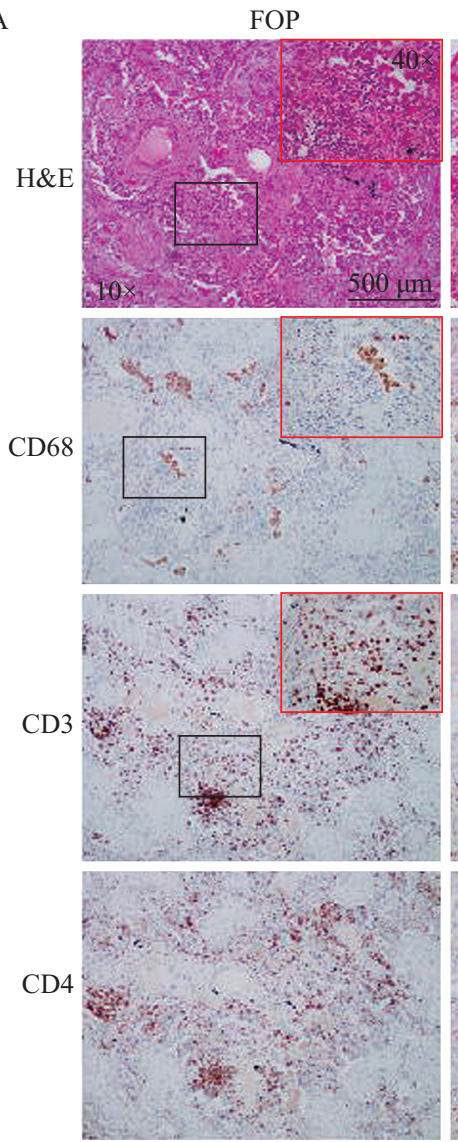

COVID-19
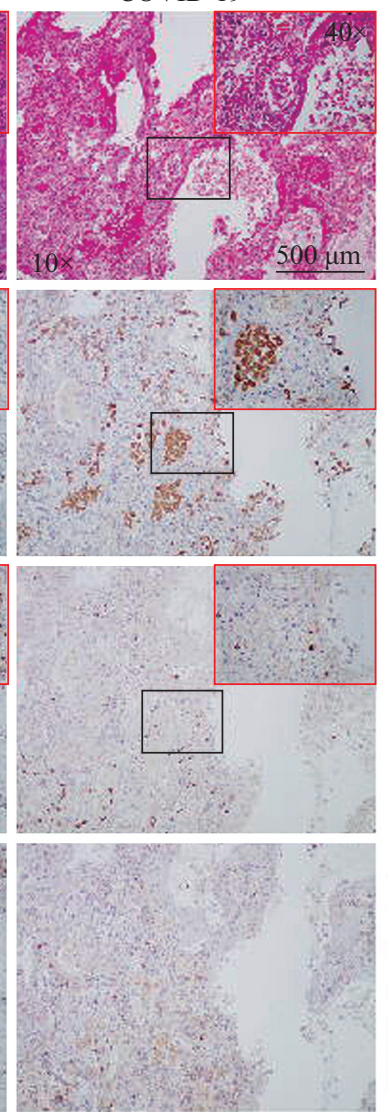

FOP
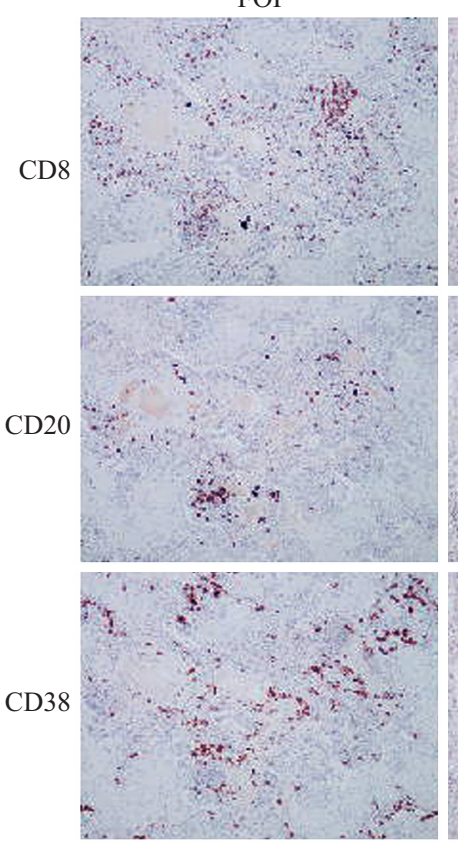

B

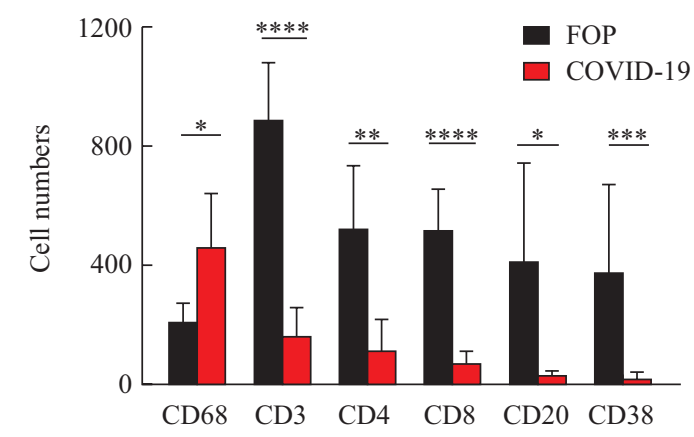

Fig. 1 Characterization of immune cells infiltrating in the lung tissues of focal organizing pneumonia (FOP) patients and deceased COVID-19 patients

A: The immune cells infiltration of postmortem COVID-19 patients was evaluated in comparison with FOP control patients by $H \& E$ staining and immunohistochemistry for detection of the immune cell-specific marker CD68 (macrophages), CD3 (total T cells), CD4 (helper T cells), CD8 (cytotoxic T cells), CD20 (total B cells), CD38 (plasma cells). B: Quantification of immune cells infiltrated within the lungs was determined by ImageJ software. Values are shown as means \pm SEM with $t$-test used. ${ }^{*} P<0.05,{ }^{* *} P<0.01,{ }^{* * *} P<0.001$ 

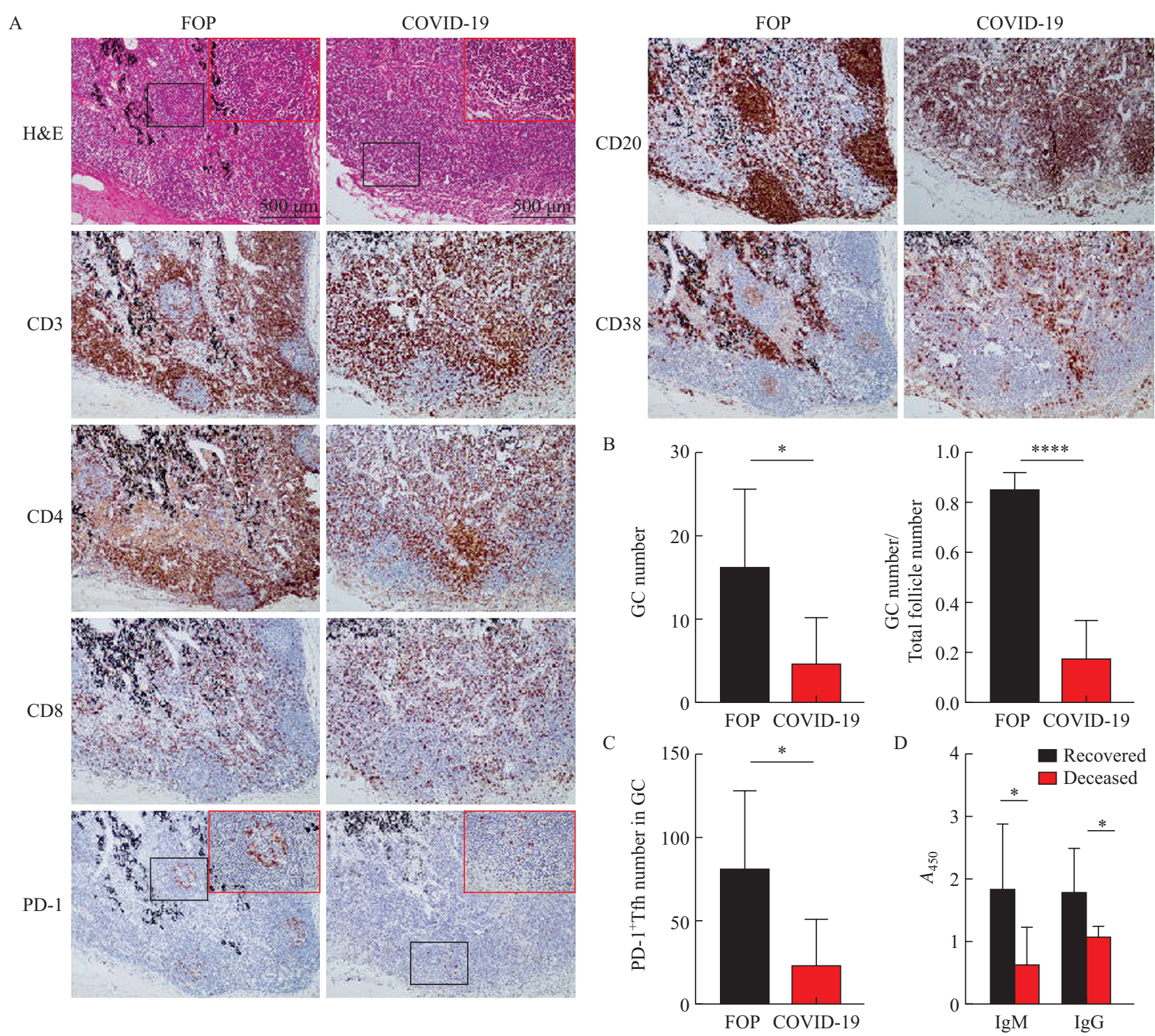

Fig. 2 Lack of adaptive immune responses in the diceased COVID-19 patients

A: H\&E staining and immunohistochemical staining of PD-1 [Tfh cells in germinal center (GC)], CD20 (total B cells), CD38 (plasma cells) in hilar lymph nodes of FOP control patients and deceased COVID-19 patients. B: GC quantification and the ratio of GC number to total follicle number were determined by immunohistochemistry. C: Tfh cells were quantified in the hilar lymph nodes of FOP control patients $(n=4)$ and deceased COVID-19 patients $(n=3)$. D: the absorbance $(A)$ values at a wavelength of $450 \mathrm{~nm}\left(A_{450}\right)$ of $\operatorname{IgM}$ and $\operatorname{IgG}$ antibodies against nucleocapsid protein and the receptor-binding domain region of the spike protein of SARS-CoV-2 in postmortem COVID-19 patients $(n=6) v s$. the convalescent COVID-19 patients $(n=8)$. Values in $\mathrm{B}, \mathrm{C}$ and $\mathrm{D}$ are shown as means \pm SEM. $t$-test. ${ }^{*} P<0.05,{ }^{* * * * *} P<0.0001$

deceased COVID-19 patients $(n=4$ FOP in control patients versus $n=6$ in COVID-19 deceased patients; fig. 2B). Similarly, Tfh cells were significantly reduced in deceased COVID-19 patients (fig. 2C, $n=3$, due to the scarcity of sample tissues), compared to FOP control patients $(n=4)$. Next we measured the IgM levels against the SARS-CoV2 S1 protein RBD and the IgG levels against SARS-CoV2 $\mathrm{N}$ protein within the recovered COVID-19 patients $(n=8)$ and the deceased COVID-19 patients ( $n=6)$. Compared to the recovered COVID-19 patients, both the IgM and IgG levels were significantly reduced within the deceased COVID-19 group (fig. 2D).

\section{DISCUSSION}

In this study, we found all the 6 deceased COVID-19 patients had significantly enhanced presence of macrophages within the lung tissues, compared to the FOP patients. Consistent with literature $^{[7,8]}$, our data suggest that macrophages are critical for the hyper-inflammation induction in the lung tissues of severe COVID-19 patients. The cytokines secreted by macrophages may mediate later apoptosis of lymphocytes. Owing to the availability of biopsy tissues, our study did not determine what kind of phenotypes of macrophages were polarized after 
SRAS-CoV2 infection.

Our data revealed a clear deficiency of Tfh cells and GC structures in secondary lymphoid organs of the deceased COVID-19 patients, indicating a profound defect of antibody affinity maturation in these deceased patients, which may have contributed to their death. By contrast, it has been reported that mildly affected and convalescent COVID-19 patients have circulating IgG, IgM, Tfh cells, virus-specific $\mathrm{CD}^{+}$ and $\mathrm{CD} 8^{+} \mathrm{T}$ cells, $\mathrm{B}$ cells, and $\mathrm{CD} 38^{+} \mathrm{CD} 8^{+} \mathrm{T}$ cells in their periphery ${ }^{[12-14]}$, indicating successful cellular and humoral immune responses in convalescent patients. It has been documented that $\mathrm{T}$ cell lymphopenia and $\mathrm{T}$ cell exhaustion occur in COVID-19 patients, which is more pronounced in the severe and critically ill patients ${ }^{[15,16]}$, yet the underlying mechanisms remain unknown. Understanding the mechanisms of SARS-CoV2 induced lymphopenia will provide key therapeutic insights for COVID-19. Furthermore, Treg cells were reduced in the severe COVID-19 patients compared to the mild cases, which might explain the exuberant pro-inflammatory responses in the severe patients due to the lack of suppression ${ }^{[17]}$.

Currently it has not been understood why these deceased patients had deficiency of the adaptive immune responses against SARS-CoV2. It could be caused by combined effects of lymphopenia, defect of Tfh differentiation and lack of appropriate GC responses. $\mathrm{Tfh}$ differentiation is a complexly regulated process involving different membrane molecules, cytokines and transcriptional factors ${ }^{[18]}$. On one hand, the substantial $\mathrm{T}$ cell and B cell lymphopenia in the severe COVID-19 patients would impair Tfh differentiation and GC responses. Additionally, both the priming to Tfh in the $\mathrm{T}$ cell zone and the $\mathrm{Tfh}$ differentiation process at the border between $\mathrm{T}$ cell and $\mathrm{B}$ cell zone could be dysregulated in these deceased patients. It would be interesting to know if the critical $\mathrm{Tfh}$ differentiation factors i.e. IL-21 or Bcl-6 expression was impaired in the deceased patients. Alternatively, considering the ubiquitous $\mathrm{T}$ cell responses and antibody production in convalescent patients ${ }^{[19]}$, the $\mathrm{T}$ cell or $\mathrm{B}$ cell repertoire diversity might be reduced in the deceased patients.

Our study has limitations on several aspects. The sample size is small due to the nature of the study. It may not fully represent all deceased COVID-19 patients in terms of immune responses in the draining lymph nodes of lung tissues. It is highly likely that a population of deceased patients had robust Tfh cell differentiation and GC formation. Furthermore, we do not have data on the viral loads within the lung tissues and hilar lymph nodes to tease out the effects of viral damages to the lung tissues. While our data argue an important protective role of adaptive immune response against SRAS-CoV2 infection, we do not know the mechanisms underlying the deficiency of
Tfh generation and GC formation in the deceased COVID-19 patients. Further pertained studies are warranted and will provide critical information on the immune responses against SARS-CoV2 and pathogenesis of COVID-19.

In summary, we found a deficiency of $\mathrm{CD}^{+}$ cytotoxic T cells, $\mathrm{CD}^{+}{ }^{+} \mathrm{T}$ cells and B cells in the lung tissues and a severe deficiency of Tfh cells and GCs in the draining hilar lymph nodes in the investigated deceased COVID-19 patients. Understanding the mechanisms of the deficiency of adaptive immune responses in these patients will be the key for the management of this epidemic.

\section{Acknowledgments}

We would like to thank Dr. Arian Laurence for critical input for the conceptualization of the project and writing of the manuscript and Dr. Ran He for critical reading of the manuscript.

\section{Conflict of Interest Statement}

The authors have declared that no conflict of interest exists.

\section{REFERENCES}

1 Huang C, Wang Y, Li X, et al. Clinical features of patients infected with 2019 novel coronavirus in Wuhan, China. Lancet, 2020,395:497-506

2 Puelles VG, Lütgehetmann M, Lindenmeyer MT, et al. Multiorgan and Renal Tropism of SARS-CoV-2. N Engl J Med, 2020,NEJMc2011400

3 Li W, Moore MJ, Vasilieva N, et al. Angiotensinconverting enzyme 2 is a functional receptor for the SARS coronavirus. Nature, 2003,426:450-4

4 Shang J, Ye G, Shi K, et al. Structural basis of receptor recognition by SARS-CoV-2. Nature, 2020,581:221224

5 Wang D, Hu B, Hu C, et al. Clinical Characteristics of 138 Hospitalized Patients with 2019 Novel Coronavirus-Infected Pneumonia in Wuhan, China. Jama, 2020,323:1061-9

$6 \mathrm{Xu}$ X, Han M, Li T, et al. Effective treatment of severe COVID-19 patients with tocilizumab. Proc Natl Acad Sci U S A, 2020,117:10970-10975

7 Giamarellos-Bourboulis EJ, Netea MG, Rovina N, et al. Complex Immune Dysregulation in COVID-19 Patients with Severe Respiratory Failure. Cell Host Microbe, 2020,27:992-1000.e3

8 Xu Z, Shi L, Wang Y, et al. Pathological findings of COVID-19 associated with acute respiratory distress syndrome. Lancet Respir Med, 2020,8:420-422

9 Qin C, Zhou L, Hu Z, et al. Dysregulation of immune response in patients with COVID-19 in Wuhan, China. Clin Infect Dis, 2020,ciaa248

10 Mesin L, Ersching J, Victora GD. Germinal Center B Cell Dynamics. Immunity, 2016,45:471-482

11 Cyster JG and Allen CDC. B Cell Responses: Cell Interaction Dynamics and Decisions. Cell, 2019,177: 524-540

12 Thevarajan I, Nguyen THO, Koutsakos M, et al. Breadth 
of concomitant immune responses prior to patient recovery: a case report of non-severe COVID-19. Nat Med, 2020,26:453-455

13 Ni L, Ye F, Cheng ML, et al. Detection of SARS-CoV-2Specific Humoral and Cellular Immunity in COVID-19 Convalescent Individuals. Immunity, 2020,52(6):971977.e3

14 Cao Y, Su B, Guo X, et al. Potent neutralizing antibodies against SARS-CoV-2 identified by high-throughput single-cell sequencing of convalescent patients' B cells. Cell, 2020,182(1):73-84.e16

15 Diao B, Wang C, Tan Y, et al. Reduction and Functional Exhaustion of T Cells in Patients with Coronavirus Disease 2019 (COVID-19). Front Immunol, 2020,11:827
16 Tan L, Wang Q, Zhang $\mathrm{D}$, et al. Lymphopenia predicts disease severity of COVID-19: a descriptive and predictive study. Signal Transduc Target Ther, 2020,5(1):33

17 Chen G, Wu D, Guo W, et al. Clinical and immunological features of severe and moderate coronavirus disease 2019. J Clin Invest, 2020,130:2620-2629

18 Crotty S. Follicular Helper CD4 T Cells (TFH). Annu Rev Immunol, 2011,29:621-663

19 Grifoni A, Weiskopf D, Ramirez SI, et al. Targets of T Cell Responses to SARS-CoV-2 Coronavirus in Humans with COVID-19 Disease and Unexposed Individuals. Cell, 2020, 181(7):1489-1501.e15

(Received July 18, 2020; accepted July 23, 2020) 